tructura y cualidades de la trompa, con su retencion indefinida. Aún en casos de permeabilidad, en que por otras causas hemos operado enfermas algún tiempo después de la histerosalpingografía y que han continuado estériles (referencia separada de los doctores Arturo Aparicio y Rodulfo Camero), se ha encontrado el medio de contraste bloqueando ovarios y trompas, transformado en una sustancia mucosa adherida por toda la pelvis. Edmundo Murray ( go, no frecuente, de granulomas pelvianos como secuela de la histerosalpingografia.

\title{
Ovaritis Poliquística Bilateral
}

La importancia que esta anomalia representa en la esterilidad matrimonial y el hecho de que su diagnóstico és casi exclusivo de la Ginecografía, hace que tratemos de ella en este estudio. A veces se piensa que se le ha dado demasiada importancia a las trompas y muy poco se le ha asignado a los ovarios cuya función fisiologica, la ovulación, es de importancia definitiva. Son varios los Test que investigan la ovulación, tales como la biopsia endometrial, temperatura basal, citología vaginal, etc., pero muy poca atención se da a los cambios en los ovarios mismos.

Guillermo Di Paola ("') insite bastante en la exploracion de los ovarios y en el estudio de los ciclos anovulatorios. Uno de los hallazgos en pacientes que consultan por esterilidad y amenorrea y que son reconozidos con poca frecuencia es la ovaritis quistica bilateral (Fig. 15)). Esta anomalia está asociadad al síndrome de Stein (11) formando la tríada principal con la esterilidad y la amenorrea, aunque esta ultima sea secundaria. Ya sabemos que las otras cáracterísticas del sindrome de Stein, como el hirsutismo, se presenta en el $50 \%$ de las pacientes y la hipoplasia uterina (Fig. 16) y de los senos, es poco frecuente.

En la pubertad de la mujer normal, bajo la influencia del estimulo pituitario, un folículo de de Graat se desarrolla hasta la madurez y luégo se rompe, liberando el primer erulo. En el sitio del foliculo roto se desarrolla el primer cuerpo amarillo. Así comienza el proceso del ciclo menstrúal con la maduración de un folículo (raramente dos) y la ruptura, repitiendose hasta la menopausia, interrumpido solamente por el embarazo.

Con ovarios poliquísticos no hay balance hormonal, pues al no romperse el folículo, se convierte en quiste de retención o la acción selectiva de la sustancia pituitaria afecta multiples folículos en vez de uno, desarrollando así una cortical con muchos quistes foliculares pequeños. En estas circunstancias, los ovarios crecen simétricamente hasta adquirir una a cuatro veces su tamano normal, permanecen ovalares. tensos y con una capsula de espesor considerable. Precisamente cuando se forman estos numerosos quistes, el epitelio germinal que es del gado, se torna fibroso y espeso sobre el sitio de la maduración del folículo. Hay fibrosis en el estroma ovárico y el contenido de los quistes es incoloro, claro, está bajo tensión y ocupa varios milímetros de diámetro. 

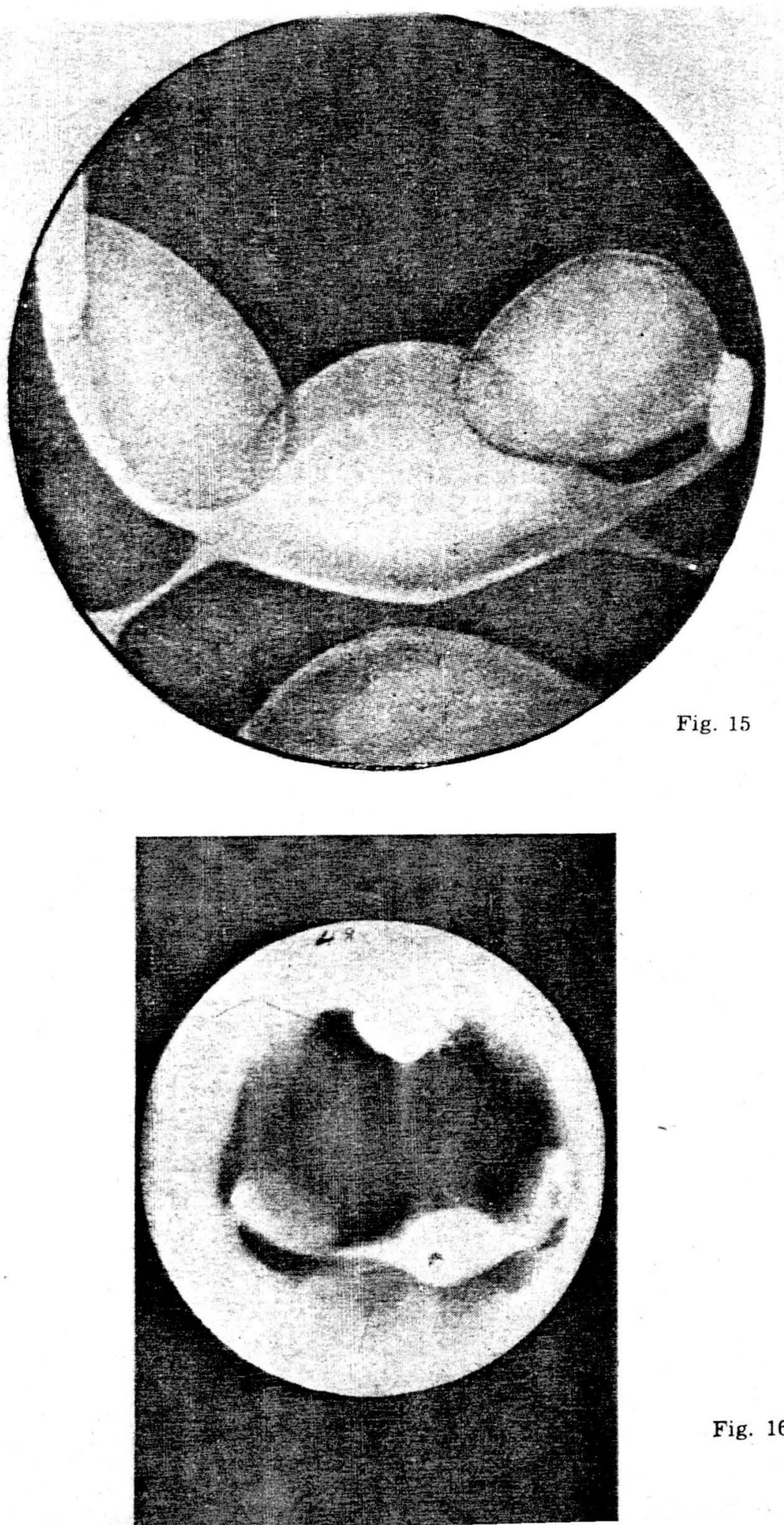

Fig. 16 
El diagnóstico se basa en algunos de los sintomas ya citados, en los hallazgos del examen pélvico, que especialmente en mujeres delgadas o con $T$. V. bajo anestesia se tocan los ovarios aumentados de tamaño; pero sobre todo, la Ginecografía y la Pelviscopia de Decker son los medios más directos y seguros para su reconocimiento hasta hacerse indispensables. El tamaño de los ovarios normales aparece en la Ginecografía como la cuarta parte del uterino; así es fácil apreciar el crecimiento cuando pasa de alli.

Pelviscopia. - Este tema ha sido ya tratado por uno de nosotros (12) y será motivo de otro trabajo que presentaremos más tarde, pero lo dẹscribimos someramente por ser tan seguro $y$ de tanta actualidad, siguiendo las instrucciones directas de su autor, el doctor Albert Decker $\left({ }^{13}\right)$, en el Knickerbocker Hospital de Nueva York en 1951. El aparato consta de un trócar y su conductor, una parte optica y su iluminación semejante a la de cualquier cistoscopio. La técnica para el uso del Pelviscopio de Decker (Culdoscopy en EE. ULI.), es la siguiente: enferma con Douglas libre, posición genu-pectoral. Se levanta la horquilla perineal con una valva, desinfeccion de los fondos de saco vaginales, se fija con ienáculos el labio posterior del cuello, traccionándolo, ya que con esta tracción se provectan los ligamentos útero-sacros que determian una foseta. Allí se punciona para anestesia de! septum con aguja de raqui y cualquier anestésico local $\because$ se introduce el trocar cuidadosamente. Al retirar el punzón y dejar el conductor, si está en la cavidad se aprecia un ruido especial producido a la entrada del aire. Por el conductor st pasa la optico del aparato y as: se pueden constatar adherencias, estado de trompas y ovarios, utero, varicoceles pelvianos, TBC peritoneal, embarazo ectopico $y$ a reces el apéndice. Teniendo el cuidado de practicar este examen en la época más probable de la ovulación, se puede constatar, si ésta se realiza, pues se verá claramante el folículo en vía de romperse o ya roto como hemos podido comprobarlo.

Trataniento. - Muchos autores y durante mucho tiempo han ensayado diversos tratamientos para los ovarios poliquisticus sin ningun resultado satisfactorio. Tan solo Kaplan (1) y otros, han tenido resultados benéficos con las radiaciones de hipófisis y orarios; por lo demás, se puede casi asegurar que no hay tratamiento médico para la ovaritis quística bilateral.

Tratamiento quirubgico. - Es el método eszogido por la mayoría de los que trabajamos en esta especialidad. El procedimiento más común es la resec ción cuneiforme; otros hacen la decapsulación parcial y todos complementan estos métodos con la puncion de los quistes restantes.

\section{Cirucia de las Trompas}

Establecida la obstrucción tubaria por la insuflación y por la histerosal. pingografia y localizada en su porción inicial, media o terminal, queda com único tratamiento la intervención quirurgica. 
En todas estas operaciones, hemos tenido como auxiliar de primer orden el insuflador de Kidde (Fig. 1). El insuflador es la guía más cierta para descubrir el canal en cualquiera de sus partes. Es indispensable que una persona entrenada en su manejo lo controle, para que el cirujano no pierda mucho tiempo durante el acto quirúrgico. Se coloca el aparato como para insuflación, antes de comenzar a operar y según el sitio obstruído se seguirán las siguientes tecnicas:

Obstrucción en la porción inicial. - Incisión de Pfanenstiell (buena para todos los casos); ya en la cavidad, se hace inspección general y se resuelve todo problema que se encuentre, dejando el propio de la trompa de último, a fin de no traumatizar la plastia hecha. Se comienza la insuflación y se repara el utero con pinzas de Alice en los ligamentos redondos; si se desea reparar la trompa que sea con Alice sin presionarla. Al llenarse la matriz con el gas, se intentará pasar un tubo de polietileno $\mathrm{N}^{0}$, del pabellón hacia la cavidad uterina para ver si el obstáculo es removible por este método. En caso negativo, se aplicará un trinquete en el segmento uterino (sonda de Nelatén $N$ i 12 que perforando los iigamentos anchos, rodea esta parte de la matriz y se fija atrás con una pinza de kelly larga) a fin de evitar la hemorragia al hacer la desinsercion e inclusión respectiva de la trompa, como lo muestran las (Figs. 17 y 18 ).

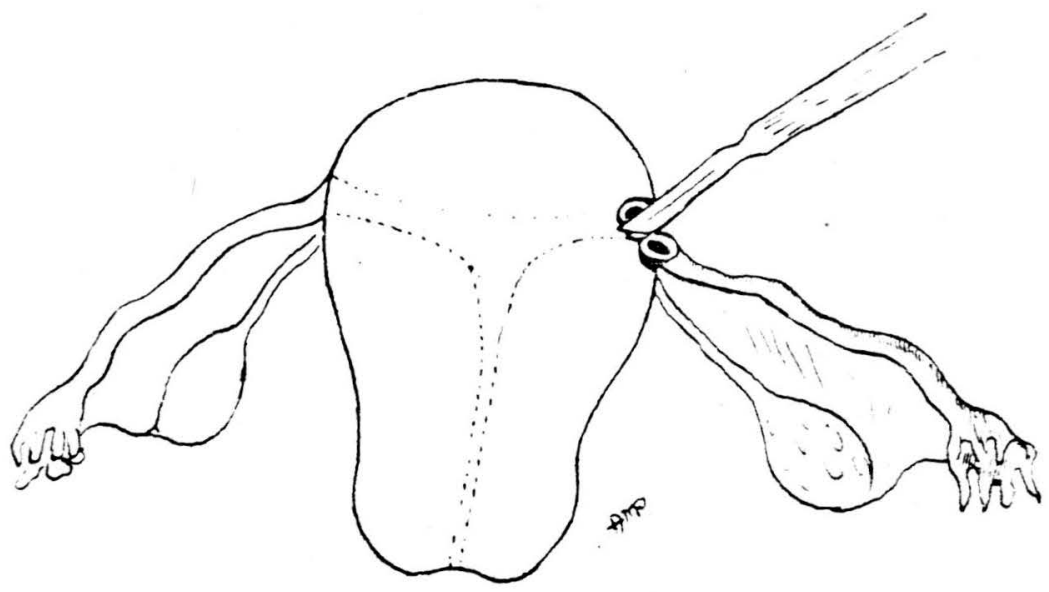




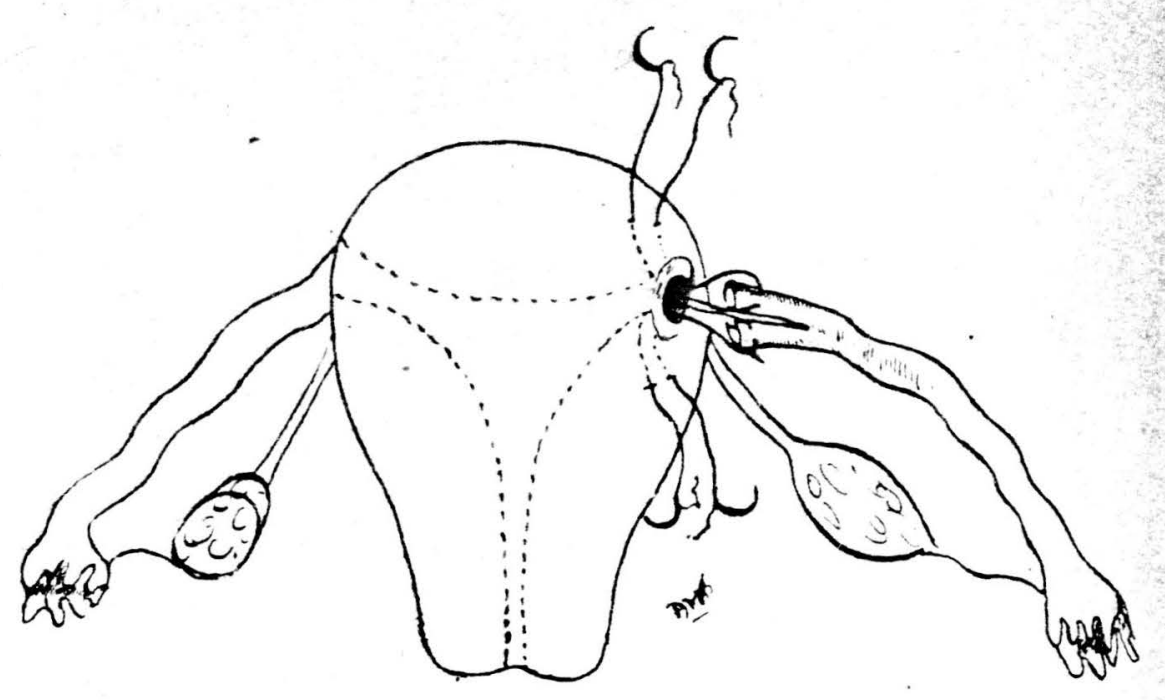

Fig 18

Las burbújas del gas servirán como guia para el paso del tubo de polietileno que se llevará hasta la vagina. fijándolo con catgut cromado $N^{0} 0$ al pabellón y a la pared vaginal. Si la obstrucción es bilateral se hará lo mismo al otro lado y en cualquier caso se dejarán los tubos de 15 a 20 días. Cuando los tubos a!. cancen la cavidad uterina, se retirará la cánula del insuflador para sacarlos a la vagina. Después de retirados los tubos se harán algunas insuflaciones para mantener la permeabilidad. Son pocos los buenos resultados que se obtienen en este caso

Obstrucción en la porción media. - Se procede como en el caso anterior sin la compresión del segmento uterino. Si el polietilenó no puede pasar del pabellón hacia la porción inicial permeable, se extirpa el trayecto obstruido y se anastomosan las porciones restantes pasando el tubo por donde aparezcan las burbújas del gas (Fig. 19) y fijándolo como en el caso anterior. Los puntos de la anastomosis término-terminal pueden ser separados y ojalá con catgut intestinal.

Obstrucción en el pabellon. - Es aquí donde el insuflador presta su máxima ayuda, ya que muestra el sitio por donde se escapan pequeñas burbújas cuando el aparato se pone a su maxima presión, o por la distención de la extrcmidad cerrada que pone de manifiesto la convergencia de los pliegues de la serosa indi- 




cando así el sitio que da acceso a la luz de la trompa. La oclusión del pabellón se caracteriza en su mavoría como lo muestran las Figs. 20 y 21 . En ambas se introduce un estilete romo por los puntos arrriba descritos y si sale gas, se introduce una pinza de Kelly y al abrirla dará salida a las fimbrias que alli estaban englobadas. Se hace ligera eversión de los bordes y se fijan con algunos puntos de catgut para evitar que vuelvan a cerrarse. En estos casos no se deja polietileno, pero se repiten las insuflaciones antes que en los casos anteriores.

Debemos hacer constar que esta clase de obstrucción tubaria la encontramos en Bogotá con mucha frecuencia; seguramente es congénita, pues a veces no la acompana ninguna lesión o secuela de lesión inflamatoria y es la más agradecida te la cirugía.
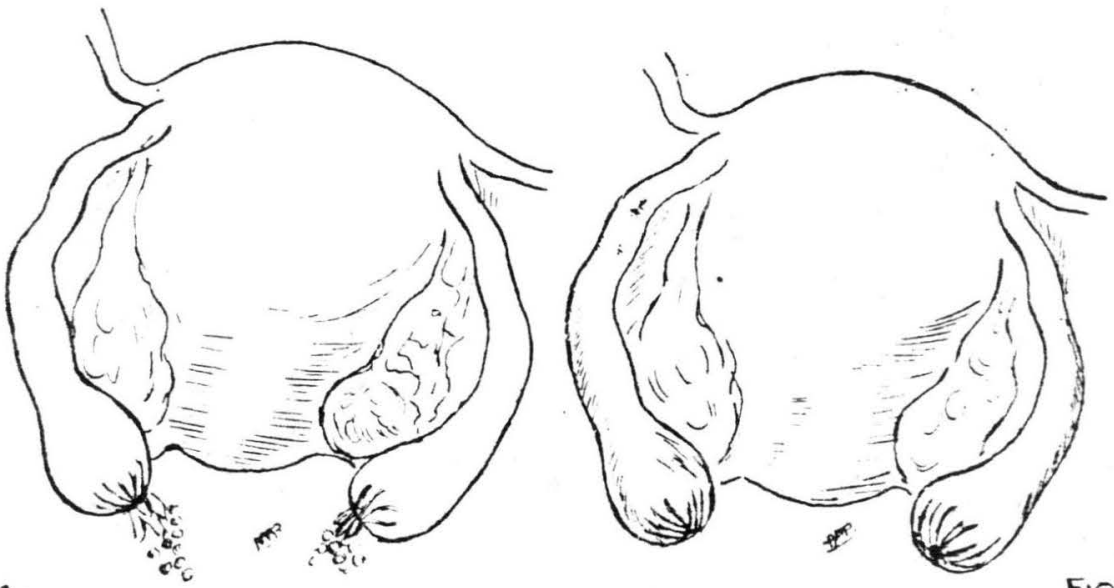

Fio 21. 
No hay casi ninguna estadística de este trabajo pues se basa en el estudio de 100 matrimonios, en un ano de iniciadas las consultas que han solicitado ayuda para resolver su problema de esterilidad, estudio incompleto por causa de los maridos que se niegan a ser examinados. Generalmente la que consulta es la mujer, fustigada por el hombre que la acusa de no darle un hijo. Otras veces implora con lágrimas ante la amenaza que le hace su cónyugue, de abandonarla o de interponer entre los dos a otra mujer. Más lastimoso aún, pero lamentablemente frecuente es cuando piden corregir su esterilidad porque el esposo las azota y las somete al hambre.

Está demostrado que más del to\% de los matrimonios estériles, lo son por causa masculina. Sin embargo el hombre en su mayoría lo sigue ignorando, y W. rechaza.

En el estudio de la pareja estéril jamás debe figurar la palabra CULPA, pues se presta a promover discusiones judiciales y por convenio se ha eliminado a fin de disimular todo estigma que pueda corresponder a cualquiera de los dos.

\section{B I B L I O G R A F I A}

1.-Peterson y Cron: "L'insufiation utero-tubaire". I. C. Rubin, 1947. Editions Medicales F. Flamarion. Paris.

2.-Rubin: "L'insuflation utero-tubaire", 1947. - 265 Editions Medicale. Flammarion, París.

3.-Hirst y Mazer, Cit. por Rubin. 1947.

4. - Novak E. and Everett H. S.. Cit. Somers H. Sturgis: "The effect of ciliary current on sperm progress in exciset human Fallopian tubes". 1947.

5. - Lamar, J. K. and Delft E.: "Cyclic penetrability of human cervical mucus to Spermatozoa in vitrio", Am. J. Phys. 129, 234, 241. - 1940.

6.-Viergiver E. and Pommerenke, W. T.: "Measurement of Cyclic variations in Quality of cervical mucous and correlation with basal temperatures", Am. J. Obst. \& Gyn. 48: 321-328. - 1944.

7.-A. Clavero Núñez: "Esterilidad Matrimonial", Salvat Editores, S. A. Barcelona, Madrid. 97-102. - 1949.

8.-Bettinotti Alberto S.: "Histerosalpingografia y Persuflación Utero-Tubárica" 1947. "El Ateneo". Bs. As.

9.-Murray E. G.: "Granulomas pelvianos persistentes, como secuela poco frecuen. te de la Histerosalpingografia". Bol. Sos. Obst. y Ginec. Bs. As., abril 1/45 Tomo IV.

10.-Di Paola Guillermo. "Conferencias sobre Esterilidad Matrimonial". 1950, Hos pital Rivadavia. Sala XIII. Bs. As.

11.-Stein, I. F.: Am. J. Obst. \& Gyn., 50: 385. - 1945.

12.-Camero C. Rodulfo: "Precauciones en la Cirugía abdómino-pélvica de la mujer Diagnóstico de precisión con la Pelviscopia". Trabajo presentado al Prime: Congreso Nacional de Cirujanos. Noviembre 1952, Bogotá.

13.-Decker Albert. Profesor de Ginecología y Obstetricia en el New York Poly clinic Medical School and Hospital.

14. -Kaplan Ira I.: Trabajo presentado al Primer Congreso Mundial para el estudi de la Esterilidad Matrimonial y al $102^{\circ}$ Congreso de la American Medical As sociation. Mayo y junio de 1953. New York. 